\title{
Outcomes after emergency abdominal surgery in COVID-19 patients at a referral center in Brazil
}

\author{
Roberto Rasslan ${ }^{1}$. Jones Pessoa dos Santos ${ }^{1} \cdot$ Carlos Augusto Metidieri Menegozzo $^{1}$ (D) \\ Alvaro Vicente Alvarez Pezzano ${ }^{1} \cdot$ Henrique Simonsen Lunardeli ${ }^{1}$. Jocielle dos Santos Miranda ${ }^{1}$. \\ Edivaldo Massazo Utiyama ${ }^{1}$. Sérgio Henrique Bastos Damous ${ }^{1}$
}

Received: 8 November 2020 / Accepted: 13 February 2021 / Published online: 24 February 2021

(C) Italian Society of Surgery (SIC) 2021

\begin{abstract}
Purpose COVID-19 is associated with high morbidity and mortality in patients undergoing surgery. Contrary to elective procedures, emergency operations should not be postponed. We aim to evaluate the profile and outcomes of COVID-19 patients who underwent emergency abdominal surgery.

Methods We performed a retrospective analysis of perioperative data of COVID-19 patients undergoing emergency surgery from April 2020 to August 2020.

Results Eighty-two patients were evaluated due to abdominal complaints, yielding 22 emergency surgeries. The mean APACHE II and SAPS were 18.7 and 68, respectively. Six patients had $\mathrm{a}_{2} \mathrm{O}_{2} / \mathrm{FiO}_{2}$ lower than 200 and more than $50 \%$ of parenchymal compromise on chest tomography.

The most common indications for emergency surgery were hernias $(6 ; 27.2 \%)$. The median length of stay was 30 days, and only two patients required reoperation. Postoperatively, $10(43.3 \%)$ patients needed mechanical ventilation for a mean of 6 days. The overall mortality rate was $31.8 \%$.

Conclusion Both postoperative morbidity and mortality are high in COVID-19 patients with respiratory compromise and abdominal emergencies.
\end{abstract}

Keywords COVID-19 · SARS-CoV-2 · Acute abdomen · Emergency surgery · Abdominal surgery · Laparoscopy

\section{Introduction}

The COVID-19 pandemic disrupted several hospital processes and forced the healthcare workers out of their routine. The surgical practice has also faced important changes due to associated risks to the patients and healthcare personnel in the COVID-19 scenario.

The higher incidence of pulmonary complications and mortality in surgical patients with COVID-19 highlighted the need to postpone elective procedures [1-3]. Several societies have issued statements guiding surgeons about which operations should be performed during the pandemic. Most

Carlos Augusto Metidieri Menegozzo

carlos.menegozzo@hc.fm.usp.br

1 Division of General Surgery and Trauma, Department of Surgery, University of Sao Paulo, Av Dr Eneas de Carvalho Aguiar, 255, Sao Paulo 05403000, Brasil institutions proceeded only with the emergency, cardiovascular and oncological surgeries [4-7].

The pandemic exacerbated the problem of delayed emergency presentation of non-COVID-19 patients. Studies have shown that the number of emergency admissions reduced during the pandemic $[8,9]$, and patients probably lingered to look for medical assistance. This delayed presentation may result in worse prognosis and should be regarded as an important healthcare problem.

Abdominal emergencies should be promptly addressed while dealing with the severity of the pulmonary disease, yielding a challenging situation for the surgical team. Emergency surgery is associated with worse outcomes in COVID-19 patients [10]. When considering elderly and frail patients, who are frequently affected by COVID-19, the risks can increase dramatically. The mortality rate can be as high as $40 \%$, compared to $23 \%$ in the same population without COVID-19 [10, 11]. 
In the COVID-19 patient, an acute abdomen may develop for multiple reasons. Although gastrointestinal symptoms are present in $39-70 \%$ of the patients, they rarely represent a critical abdominal condition. However, the physiopathological impact of COVID-19 may increase the risks of an acute abdomen. Up to $4 \%$ of the patients may develop mesenteric ischemia, which can be related to SARS-Cov-2 coagulopathy $[12,13]$, while respiratory symptoms may predispose abdominal hernias to incarceration. Moreover, critical patients may develop abdominal conditions such as acute cholecystitis, hollow viscus perforations, and intestinal pseudo-obstruction (Ogilvie's Syndrome).

This study aims to evaluate COVID-19 patients who developed acute abdominal conditions and required emergency surgery, focusing on postoperative morbidity and mortality.

\section{Methods}

This is a retrospective study using prospectively collected data from patients admitted between April 2020 and August 2020. The Ethics Committee approved this study under the number 4.107.232. This study is being reported according to the STROBE criteria [14].

As part of the action plan to deal with the pandemic, the Hospital das Clínicas of the Universidade de São Paulo has dedicated over 500 ward beds and 300 ICU beds for COVID19 patients. As a result, several complex cases of the State of São Paulo were referred to our care, and the Division of General Surgery and Trauma organized a dedicated team to deal with the abdominal emergencies in COVID-19 patients.

All patients undergoing abdominal emergency surgery were included. The following variables were analyzed: the demographic profile, ASA score, disease severity, ventilation support, perioperative data, complications, length of stay, and mortality. We defined emergency surgery as procedures performed in the first $24 \mathrm{~h}$ after surgical indication. Disease severity was evaluated using APACHE II and SAPS 3 scores and the extent of lung compromise by computerized tomography (CT), which was classified in $<25 \%, 25-50 \%$, and $>50 \%$ [15]. Preoperative respiratory support variables included $\mathrm{PaO}_{2} / \mathrm{FiO}_{2}$ and the need for mechanical ventilation. Perioperative data included diagnosis, type of surgery, operative risk scores (National Surgical Quality Improvement Program-NSQIP, and Physiological and Operative Severity Score for Enumeration of Mortality and Morbidity-pPOSSUM) [16, 17].

Surgical postoperative complications were graded according to the Clavien-Dindo classification [18]. Mortality was considered during hospitalization and did not encompass follow-up data.

The statistical analysis provided descriptive statistics, which were depicted as mean, standard deviation, and percentage for the continuous variables and median and range for categorical parameters.

\section{Results}

Our group performed 82 surgical consults during the study period, resulting in $22(26.8 \%)$ emergency surgeries. All cases were COVID-19 confirmed by a reverse-transcriptase polymerase chain reaction. Table 1 depicts the profile of the studied patients. Twenty patients $(90.9 \%)$ had at least one comorbidity, while $9(40.9 \%)$ had three or more. $\mathrm{PaO}_{2} /$ $\mathrm{FiO}_{2}$ was lower than $200 \mathrm{mmHg}$ in six patients (31.5\%). Ten patients $(45 \%)$ required mechanical ventilation preoperatively for a mean time of 11 days, and seven patients (31.8\%) were still intubated at the time of surgical indication.

The data regarding emergency surgical procedures are listed in Table 2. Of the six hernia repairs, there were three ventral, one femoral, one inguinal, and one incisional. The femoral hernia required bowel resection. Five patients were referred to our service with postoperative complications from emergency surgeries who developed SARS-CoV-2 infection. There were two cases of postoperative fascial dehiscence. The first was in a patient who underwent left colectomy due to obstructive colon cancer, and the second was after a right colectomy due to ischemic colitis. We had two cases of anastomotic leakage, one related to a colonic anastomosis after a multivisceral resection of pancreatic cancer, and another due to an iatrogenic duodenal injury during nephrectomy for renal abscess. The patient with the ovarian cyst rupture was reoperated by laparoscopy due to recurrent bleeding. Regarding the mesenteric ischemia, the two cases were submitted to a damage control procedure and a subsequent "second-look" and in just one, the intestinal anastomosis was performed. The sole case of small bowel obstruction was due to an actinic stricture after radiotherapy for cervix cancer, which required an enterectomy. We had one case of splenic abscess after cardiac surgery to treat complicated endocarditis. Overall, laparoscopy was performed in 5 patients $(22.7 \%)$.

Table 3 depicts the postoperative data of the studied patients. We observed four cases of grade III complications, namely an intraperitoneal hemorrhage treated with surgery, gastrointestinal bleeding evaluated by enteroscopy, a postcholecystectomy biliary fistula, and an anastomotic leakage treated by percutaneous drainage followed by reoperation. Of the three grade $\mathrm{V}$ complications, two were related to gastrointestinal bleeding, which probably does not directly associate with the emergency procedure. The other fatality 
Table 1 Profile of COVID-19 patients undergoing emergency abdominal surgery

\begin{tabular}{|c|c|}
\hline Parameter & $n=22$ \\
\hline Age (Median, range) & $62(16-91)$ \\
\hline \multicolumn{2}{|l|}{ Gender } \\
\hline Male & $12(56.5 \%)$ \\
\hline Female & $10(43.5 \%)$ \\
\hline Body mass index $\left(\mathrm{kg} / \mathrm{m}^{2}\right)($ mean $\pm \mathrm{SD})$ & 25.8 \\
\hline No. of comorbidities (median, range) & $2(0-5)$ \\
\hline 0 & $2(9 \%)$ \\
\hline 1 & $6(27.2 \%)$ \\
\hline 2 & $5(22.7 \%)$ \\
\hline$>3$ & $9(40.9 \%)$ \\
\hline \multicolumn{2}{|l|}{ Comorbidities } \\
\hline Hypertension & $11(50 \%)$ \\
\hline Diabetes & $10(45.4 \%)$ \\
\hline Obesity & $4(18.2 \%)$ \\
\hline Smoking & $3(13.6 \%)$ \\
\hline Cancer & $3(13.6 \%)$ \\
\hline Arrhythmia & $2(9.1 \%)$ \\
\hline COPD & $1(4.6 \%)$ \\
\hline \multicolumn{2}{|l|}{ ASA score } \\
\hline 1 & $2(9.1 \%)$ \\
\hline 2 & $5(22.7 \%)$ \\
\hline 3 & $10(45.4 \%)$ \\
\hline 4 & $5(22.7 \%)$ \\
\hline \multicolumn{2}{|l|}{ Hospitalization at the surgical indication } \\
\hline Intensive care unit & $9(39.1 \%)$ \\
\hline Ward or emergency department & $11(60.9 \%)$ \\
\hline \multicolumn{2}{|l|}{ Disease severity } \\
\hline SAPS3 & $66(40-113)$ \\
\hline APACHE 2 & $17(8-38)$ \\
\hline Mechanical ventilation at the surgery indication & $7(32 \%)$ \\
\hline Days of mechanical ventilation (median, range) & $6(1-20)$ \\
\hline \multicolumn{2}{|l|}{ Ratio of $\mathrm{PaO}_{2}$ to $\mathrm{FiO}_{2}, \mathrm{mmHg}$} \\
\hline$>300$ & $11(50 \%)$ \\
\hline $200-300$ & $5(23 \%)$ \\
\hline $100-200$ & $2(9 \%)$ \\
\hline$<100$ & $4(18 \%)$ \\
\hline \multicolumn{2}{|l|}{ Chest tomography severity score } \\
\hline$<25 \%$ & $6(27.2 \%)$ \\
\hline $25-50 \%$ & $10(45.4 \%)$ \\
\hline$>50 \%$ & $6(27.2 \%)$ \\
\hline
\end{tabular}

was due to complications associated with an enteroatmospheric fistula.

Our study's mortality rate was $31.8 \%$, and the details of the patients who died are exhibited in Table 4.

\section{Discussion}

The present study analyzed patients who were hospitalized due to COVID-19 respiratory compromise and developed emergency abdominal conditions. The severity of such patients can be demonstrated preoperatively by the elevated APACHE II and SAPS 3 scores and the prevalence of mechanical respiratory support in $45.4 \%$ of the patients. Postoperatively, such severity is also represented by the need for mechanical ventilation in $43 \%$ of the patients and the mortality rate of $31.8 \%$.

Only a few reports have specifically evaluated emergency abdominal surgery outcomes in hospitalized patients already under treatment for COVID-19 respiratory failure. Seeliger et al. described six patients, $50 \%$ of which developed acute respiratory distress syndrome (ARDS), and 33\% died [19]. The same study also showed that, in seven patients with acute abdomen and SARS-CoV-2 coinfection with mild or no respiratory compromise, mortality was $14.3 \%$, while $28.5 \%$ developed postoperative pulmonary complications. These results highlight the role that the severity of the COVID-19 pulmonary disease may play in emergency surgical patients.

There are three distinct situations regarding COVID19 infection and abdominal emergency surgical patients. The first is developing an acute condition during hospitalization due to complications of COVID-19; secondly, the abdominal condition may arise in an asymptomatic or oligosymptomatic COVID-19 patient; lastly, an emergency surgical patient may acquire COVID-19 in the postoperative period. The exact pathogenic pathway of the abdominal complications is not clearly determined in such patients. Whether the acute abdomen results from the SARS-Cov-2 infection, the decompensation of comorbidities, or the treatment of a critically ill patient is not well understood. The high incidence of thrombotic complications in the initial reports led to discovering the thrombogenic effect of the COVID-19 inflammation [20]. Therefore, mesenteric thrombosis may be attributed to the impact of the infection. Kaafarani et al. [10] and Ignat et al. [21] described seven mesenteric ischemia cases, three of which were venous thrombosis. In our study, the two mesenteric ischemia cases were probably related to the illness severity and the comorbidities resulting in arterial thrombosis.

The abdominal wall complications might be an indirect result of COVID-19, in the sense that respiratory symptoms and complications may lead to increased intraabdominal pressures. According to Guan et al. [22], 67.8\% of the infected patients developed coughing. Our study showed that $35 \%$ of the emergency abdominal operations were due to abdominal wall complications, six due to 
Table 2 Diagnosis and surgical procedure of 22 COVID-19 patients undergoing emergency surgery

Table 3 Morbidity and mortality of 22 COVID19 patients who underwent emergency abdominal surgery

\begin{tabular}{lll}
\hline Diagnosis & Procedure & $N$ \\
\hline Hernia & Hernia repair with mesh & $6(27.2 \%)$ \\
Acute cholecystitis & Laparoscopic Cholecystectomy & $2(9.1 \%)$ \\
Acute appendicitis & Laparoscopic Appendectomy & $2(9.1 \%)$ \\
Reoperation & & \\
$\quad$ Abdominal fascial dehiscence & Fascial suture with onlay mesh & $2(9.1 \%)$ \\
Intestinal fistula & Laparotomy and suture & $2(9.1 \%)$ \\
Mesenteric ischemia & Intestinal resection + damage control & $2(9.1 \%)$ \\
Ogilvie's syndrome & Right colectomy & $2(9.1 \%)$ \\
& Loop colostomy & $1(4.5 \%)$ \\
Intestinal obstruction & Laparotomy & $1(4.5 \%)$ \\
Splenic abscess & Splenectomy & $1(4.5 \%)$ \\
Ruptured ovarian cyst & Laparoscopy for hemostasis & $1(4.5 \%)$ \\
\hline
\end{tabular}

Table 4 Profile of the COVID-19 patients who died after the surgical procedure

\begin{tabular}{|c|c|c|c|c|c|c|c|c|}
\hline Patient & Age & Gender & ASA score & $\begin{array}{l}\text { SAPS3/ } \\
\text { APACHEII }\end{array}$ & $\begin{array}{l}\mathrm{PaO}_{2} / \\
\mathrm{FiO}_{2} \\
\mathrm{mmHg}^{2}\end{array}$ & $\begin{array}{l}\text { Pulmonary } \\
\text { severity (CT } \\
\text { Scan) }\end{array}$ & Diagnosis & Surgery \\
\hline 1 & 79 & $\mathrm{~F}$ & 3 & $76 / 25$ & 67 & $\leq 25$ & Strangulated femoral hernia & Inguinotomy + enterectomy \\
\hline 2 & 91 & M & 2 & $56 / 11$ & 223 & $25-50 \%$ & Epigastric hernia & Hernia repair \\
\hline 3 & 62 & $\mathrm{~F}$ & 4 & $62 / 26$ & 385 & $\geq 50 \%$ & Umbilical hernia & Hernia repair \\
\hline 4 & 52 & $\mathrm{~F}$ & 2 & $61 / 36$ & 97 & $\geq 50 \%$ & $\begin{array}{l}\text { Fascial dehiscence (posopera- } \\
\text { tive of colon resection-colon } \\
\text { câncer) }\end{array}$ & Fascial suture with onlay mesh \\
\hline 5 & 57 & M & 3 & $73 / 19$ & 70 & $\leq 25$ & Intestinal fistula & Laparotomy \\
\hline 6 & 81 & M & 4 & $113 / 31$ & 125 & $\geq 50$ & Mesenteric ischememia & $\begin{array}{l}\text { Intestinal ressection + damage } \\
\text { control }\end{array}$ \\
\hline 7 & 61 & M & 3 & $65 / 38$ & 667 & $25-50 \%$ & $\begin{array}{l}\text { Complicated Ogilvie's Syn- } \\
\text { drome }\end{array}$ & Right colectomy \\
\hline
\end{tabular}


incarcerated hernias, and two fascial dehiscence. When analyzing non-COVID-19 patients, the mortality of incarcerated hernias in elderly patients is not despicable. Arenal et al. [23] observed that 5 and $16 \%$ of octogenarians and nonagenarians died, respectively, due to such a condition.

Moreover, Nilsson et al. [24] demonstrated a mortality rate of $7.1 \%$ after emergency surgery due to femoral hernias in patients over 60 years. In our study, the mortality rate of incarcerated hernias was 50\%, which can be explained by the severity of the respiratory compromise due to COVID-19, the presence of intestinal ischemia, and the higher surgical risk in a patient with advanced cirrhosis and a nonagenarian. Hence, our results highlight that when we add the complications of COVID-19 to an already severe abdominal complication, the result is worse.

During the pandemic, several authors and societies issued statements regarding nonoperative treatment of conditions such as acute appendicitis and cholecystitis due to the risk of contamination and the potential for worse outcomes [25]. Gao et al. [26] suggested that, despite the elevated morbidity and mortality rates in COVID-19 patients, abdominal conditions should be surgically addressed promptly. In line with that opinion, we decided to operate on two patients with grade 2 acute cholecystitis, both of which had gallbladder necrosis.

Another issue of controversy is the laparoscopic approach and its risk of aerosolization. Coccolini et al. [27] demonstrated the virus's presence in the peritoneal fluid, showing that the risk of contamination during the procedure is real. For this reason, some societies believe that laparoscopic indication should be judicious and selective. We believe that in centers with experience and resources, especially a gas filter device, this minimally invasive access may have the same indication in non-COVID patients. In the present study, the cholecystectomies, appendectomies, and reoperation due to the ovarian bleeding were performed by laparoscopy. However, due to the small number of laparoscopic procedures, we cannot provide any recommendations based on our results.

While early diagnosis of abdominal complications in COVID-19 critically ill patients is of utmost importance, their clinical condition in the ICU, including the need for mechanical ventilation and prone positioning, renders early diagnosis a significant challenge. In our cohort, Ogilvie's syndrome exemplifies the importance of timely diagnosis in preventing associated complications. Out of three cases, two already exhibited cecum necrosis, perforation, and peritonitis, resulting in intestinal resection.

Postoperative mortality of COVID-19 patients undergoing abdominal surgery is high, even in asymptomatic patients or those who contracted the infection in the postoperative period. The COVIDSurg Collaborative reported a $23.1 \%$ mortality rate, including $25 \%$ of elective cases and patients with postoperative SARS-Cov-2 infection [8]. This multicenter international study [8], including 1128 patients undergoing surgery, showed higher mortality after an emergency (26\%) when compared to elective procedures (19\%). Moreover, Lei et al. [2] observed $20.5 \%$ of deaths after elective surgeries during the incubation period of COVID-19 infection.

Abdominal emergency surgery results in a mortality rate of $7.2 \%$ and $26.8 \%$ worldwide for ASA 3 and ASA 4 patients, respectively [28]. The mortality rate of our study was higher (31.8\%). Interestingly, when surgical risk was calculated using NSQIP and p-POSSUM, results were 17\% and $25.2 \%$, respectively. Therefore, those scores underestimated the mortality risk in our surgical population. We speculate that COVID-19 adds further morbidity that is not quantifiable by those risk scores, a hypothesis that is supported by the disease severity calculators used in our study. The APACHE II and SAPS 3 scores were 17 and 66, respectively. While in South America, the mortality rate associated with a mean SAPS 3 score reported in this study is $64.7 \%$, in our study, $31.8 \%$ of the patients died. These findings underscore the weight of the severity of the disease in determining the patients' outcomes.

Our study has limitations, one of which is its retrospective nature. Secondly, the diversity of abdominal surgeries performed may have impacted the outcome, as different diseases and procedures are not always comparable. However, we highlight that all included COVID-19 patients were critically ill, demonstrated by the severity scores. Also, despite the emergency condition, take-back cases coming from other institutions should be compared cautiously with primary surgical patients. We also acknowledge the difficulty in differentiating if the patients' severity is primarily due to the COVID-19 infection or the abdominal condition. The identification of risk factors for postoperative complications and mortality would be interesting, however, due to the small number of patients in our study, it was not possible to perform uni- or multi-variate analysis. Despite the limited number of included patients, this case series represents a single COVID-19 dedicated group's experience during the pandemic. As far as we know, this is the largest report from a single group regarding abdominal emergency surgery in hospitalized patients with severe COVID-19 with respiratory compromise.

\section{Conclusion}

COVID-19 patients with severe respiratory distress exhibit high morbidity and mortality when subjected to emergency surgery due to acute abdominal conditions. Prompt evaluation and treatment by a dedicated surgical team may be responsible for a mortality rate lower than predicted. 
Supplementary Information The online version contains supplementary material available at https://doi.org/10.1007/s13304-021-01007-5.

Funding This research did not receive any specific grant from funding agencies in the public, commercial, or not-for-profit sectors.

Data availability Data will be available for people who contact the authors.

\section{Compliance with ethical standards}

Conflict of interest The authors declare not having any conflict of interests related to this study.

Ethical approval The Ethics Committee approved this study under the number 4.107.232.

\section{References}

1. Nahshon C, Bitterman A, Haddad R, Hazzan D, Lavie O (2020) Hazardous postoperative outcomes of unexpected COVID-19 infected patients: a call for global consideration of sampling all asymptomatic patients before surgical treatment. World J Surg 44(8):2477-2481

2. Lei S, Jiang F, Su W, Chen C, Chen J, Mei W et al (2020) Clinical characteristics and outcomes of patients undergoing surgeries during the incubation period of COVID-19 infection. E Clin Med 21:100331

3. Aminian A, Safari S, Razeghian-Jahromi A, Ghorbani M, Delaney CP (2020) COVID-19 outbreak and surgical practice: unexpected fatality in perioperative period. Ann Surg 272(1):e27-e29

4. Moletta L, Pierobon ES, Capovilla G, Costantini M, Salvador R, Merigliano $S$ et al (2020) International guidelines and recommendations for surgery during Covid-19 pandemic: a systematic review. Int J Surg 79:180-188

5. Cancer patient management during the COVID-19 pandemic? ESMO (2020) https://www.esmo.org/guidelines/cancer-patientmanagement-during-the-covid-19-pandemic

6. ACS, Clinical issues and guidance (2020) https://www.facs.org/ covid-19/clinical-guidance

7. Qadan M, Hong TS, Tanabe KK, Ryan DP, Lillemoe KD (2020) A multidisciplinary team approach for triage of elective cancer surgery at the Massachusetts general hospital during the novel coronavirus COVID-19 outbreak. Ann Surg 272(1):e20-e21

8. Cano-Valderrama O, Morales X, Ferrigni CJ, Martín-Antona E, Turrado V et al (2020) Acute care surgery during the COVID-19 pandemic in Spain: changes in volume, causes and complications. A multicentre retrospective cohort study. Int J Surg 80:57-161

9. Pereira X, Lima DL, Moran-Atkin E, Malcher F (2020) Where have the surgical patients gone during the COVID-19 pandemic? Rev Col Bras Cir 47:e20202733

10. Collaborative C (2020) Mortality and pulmonary complications in patients undergoing surgery with perioperative SARS-CoV-2 infection: an international cohort study. Lancet 396(10243):27-38

11. Team NP (2018) Fourth patient report of the National Emergency Laparotomy Audit
12. Kaafarani HMA, El Moheb M, Hwabejire JO, Naar L, Christensen MA, Breen K et al (2020) Gastrointestinal complications in critically Ill patients with COVID-19. Ann Surg 272(2):e61-e62

13. Zhang JJ, Dong X, Cao YY, Yuan YD, Yang YB, Yan YQ et al (2020) Clinical characteristics of 140 patients infected with SARS-CoV-2 in Wuhan. China Allergy 75(7):1730-1741

14. von Elm E, Altman DG, Egger M, Pocock SJ, Gøtzsche PC, Vandenbroucke JP et al (2007) The Strengthening the Reporting of Observational Studies in Epidemiology (STROBE) statement: guidelines for reporting observational studies. Lancet 370(9596): 1453-1457

15. Yuan M, Yin W, Tao Z, Tan W, Hu Y (2020) Association of radiologic findings with mortality of patients infected with 2019 novel coronavirus in Wuhan, China. PLoS ONE 15(3):e0230548

16. Bilimoria KY, Liu Y, Paruch JL, Zhou L, Kmiecik TE, Ko CY et al (2013) Development and evaluation of the universal ACS NSQIP surgical risk calculator: a decision aid and informed consent tool for patients and surgeons. J Am Coll Surg 217(5):833-842

17. Copeland GP, Jones D, Walters M (1991) POSSUM: a scoring system for surgical audit. Br J Surg 78(3):355-360

18. Dindo D, Demartines N, Clavien PA (2004) Classification of surgical complications: a new proposal with evaluation in a cohort of 6336 patients and results of a survey. Ann Surg 240(2):205-213

19. Seeliger B, Philouze G, Cherkaoui Z, Felli E, Mutter D, Pessaux $\mathrm{P}$ (2020) Acute abdomen in patients with SARS-CoV-2 infection or co-infection. Langenbecks Arch Surg 405(6):861-866

20. Connors JM, Levy JH (2020) COVID-19 and its implications for thrombosis and anticoagulation. Blood 135(23):2033-2040

21. Ignat M, Philouze G, Aussenac-Belle L, Faucher V, Collange O, Mutter D et al (2020) Small bowel ischemia and SARS-CoV-2 infection: an underdiagnosed distinct clinical entity. Surgery 168(1):14-16

22. Guan WJ, Ni ZY, Hu Y, Liang WH, Ou CQ, He JX et al (2020) Clinical characteristics of coronavirus disease 2019 in China. N Engl J Med 382(18):1708-1720

23. Arenal JJ, Rodríguez-Vielba P, Gallo E, Tinoco C (2002) Hernias of the abdominal wall in patients over the age of 70 years. Eur J Surg 168(8-9):460-463

24. Nilsson H, Stylianidis G, Haapamäki M, Nilsson E, Nordin P (2007) Mortality after groin hernia surgery. Ann Surg 245(4):656-660

25. De Simone B, Chouillard E, Di Saverio S, Pagani L, Sartelli M, Biffl WL et al (2020) Emergency surgery during the COVID-19 pandemic: what you need to know for practice. Ann R Coll Surg Engl 102(5):323-332

26. Gao Y, Xi H, Chen L (2020) Emergency surgery in suspected COVID-19 patients with acute abdomen: case series and perspectives. Ann Surg 272(1):e38-e39

27. Coccolini F, Tartaglia D, Puglisi A, Giordano C, Pistello M, Lodato $\mathrm{M}$ et al (2020) SARS-CoV-2 is present in peritoneal fluid in COVID-19 patients. Ann Surg 272(3):e240-e242

28. Collaborative G (2016) Mortality of emergency abdominal surgery in high, middle and low-income countries. Br J Surg 103(8):971-988

Publisher's Note Springer Nature remains neutral with regard to jurisdictional claims in published maps and institutional affiliations. 